\title{
Auditory Brainstem Responses in Newborns With Down Syndrome at a Japanese Perinatal Center
}

\author{
Mieko Sato $^{a}$, Shunji Suzukia, b
}

\begin{abstract}
Background: Although many children with Down syndrome experience hearing loss, there have been little researches to investigate auditory brainstem responses (ABRs) in newborns with Down syndrome.
\end{abstract}

Methods: We examined ABRs in 24 Japanese newborns with Down syndrome at $20.6 \pm 6.0$ days after delivery compared with sex-, age- and weight-matched Japanese newborns without Down syndrome as control.

Results: Wave I component latency (CL) was longer in ABRs of infants with Down syndrome than control newborns, while wave $\mathrm{V}$ $\mathrm{CL}$ and the wave III-V and wave I-V interpeak latencies were shorter in ABRs of infants with Down syndrome than control newborns.

Conclusions: The current results indicate the presence of developmental anomaly of the inner ear in newborns with Down syndrome.

Keywords: Down syndrome; Auditory brainstem response; Newborn

\section{Introduction}

Undetected congenital permanent hearing loss impairs development in infants by diminishing their ability to acquire language and cognitive skills at an appropriate age [1]. All Down syndrome children have different degrees of devel-

\footnotetext{
Manuscript accepted for publication September 11, 2014

a Department of Obstetrics and Gynecology, Japanese Red Cross Katsushika Maternity Hospital, Tokyo, Japan

${ }^{\mathrm{b}}$ Corresponding Author: Shunji Suzuki, Department of Obstetrics and Gynecology, Japanese Red Cross Katsushika Maternity Hospital,

5-11-12 Tateishi, Katsushika-ku, Tokyo 124-0012, Japan.

Email: czg83542@mopera.ne.jp
}

doi: http://dx.doi.org/10.14740/ijcp166w opmental disabilities, developmental delay and developmental brain abnormalities associated with central myelination delay and cortical dysgenesis [2, 3]; however, conductive hearing loss has been reported to be very common in Down syndrome $[4,5]$. In an earlier study of 45 infants with Down syndrome by Raut et al [4], for example, the incidence of hearing loss in the first year of life with Down syndrome was high (34\%) by Universal Newborn Hearing Screening (UNHS). Eighty-five percent of them were observed to be conductive hearing loss with $64 \%$ in mild-moderate range. One-third of the hearings were normalized after treatment, one-third remained unaltered and one-third of infants did not attend follow-up. In another examination by Park et al [5], on the other hand, 87 (26\%) of 332 infants with Down syndrome infants did not pass the UNHS. Thirty-three of these children $(38 \%)$ had a conductive hearing loss attributed to serous otitis media. Five infants had sensorineural hearing loss; three children were diagnosed with a mixed hearing loss. More than $43 \%$ of the newborns with Down syndrome who passed the UNHS developed a conductive hearing loss requiring insertion of ventilation tubes.

Using auditory brainstem response (ABR), which is an effective noninvasive measure of sensorineural and conductive hearing loss, conductive hearing is typically indicated to be a delayed wave I component latency (CL) or a poorly defined wave I [6]. In ABR, wave I and wave II are generated from the eighth nerve and cochlea on the ipsilateral side, while waves III and V are generated from a complex interaction of both contralateral and ipsilateral brainstem anatomy. In an earlier report examining ABRs in American newborns with Down syndrome at $3.3 \pm 2.2$ days after delivery by Kitter et al [7], wave III and wave V CLs and the wave I-III interpeak latency (IPL) were shorter in ABRs of 15 infants with Down syndrome than in sex-, age- and weight-matched 15 normal newborns, while the wave III-V IPL was not different. Their results indicate the presence of anomalies in the lower rather than upper brainstem auditory pathways in Down syndrome [7]. Although many children with Down syndrome experience hearing loss, there have been other little researches to investigate ABRs in newborns with Down syndrome.

In the current study, therefore, we examined ABRs in 
Table 1. Mean CLs and IPLs in Neonates With and Without Down Syndrome

\begin{tabular}{lllc}
\hline & $\begin{array}{l}\text { Down syndrome }(\mathbf{n}=\mathbf{2 3}, \mathbf{n i n e} \\
\text { female })\end{array}$ & Control $(\mathbf{n}=\mathbf{2 3}$, nine female $)$ & P-value \\
\hline Wave I CL & $1.55 \pm 0.22$ & $1.47 \pm 0.13$ & 0.049 \\
Wave III CL & $4.33 \pm 0.44$ & $4.36 \pm 0.26$ & 0.408 \\
Wave V CL & $6.40 \pm 0.34$ & $6.61 \pm 0.29$ & 0.114 \\
Wave I-III IPL & $2.80 \pm 0.35$ & $2.90 \pm 0.20$ & 0.024 \\
Wave III-V IPL & $2.06 \pm 0.38$ & $2.25 \pm 0.24$ & $<0.001$ \\
Wave I-V IPL & $4.85 \pm 0.31$ & $5.15 \pm 0.22$ & 0.13 \\
\hline
\end{tabular}

Data are presented as mean \pm SD. CL: component latency; IPL: interpeak latency.

Japanese newborns with Down syndrome at $20.6 \pm 6.0$ days after delivery compared with sex-, age- and weight-matched Japanese newborns without Down syndrome at Japanese Red Cross Katsushika Maternity Hospital, Tokyo, Japan.

\section{Patients and Methods}

The protocol for this study was approved by the Ethics Committee of the Japanese Red Cross Katsushika Maternity Hospital. Our hospital is one of the major perinatal centers for high risk pregnancies in Tokyo, Japan.

We examined the 24 (nine female) Japanese infants with Down syndrome who were born at Japanese Red Cross Katsushika Maternity Hospital from 2011 to 2013. All examinations were performed by one experienced examiner (M.S.). After obtaining the family's consents, in the current study infants were screened with a Neuropack $\mu^{\circledR}$, an ABR sensor at $20.6 \pm 6.0$ days after delivery. Infants who did not pass the ABR were referred to the specialists of hearing loss as previously reported [8]. We also examined the sex-, age- and weight-matched Japanese newborns without Down syndrome as control.

Data are presented as mean $\pm \mathrm{SD}$. The Student's $t$-test for continuous variables was used. Differences with $\mathrm{P}<0.05$ were considered significant.

\section{Results}

One (4\%) male infant with Down syndrome who was delivered at 37.4 weeks' gestation weighing 2,506 g did not pass the ABR stimuli $105 \mathrm{~dB}$, and he was referred to the specialists of hearing loss. Therefore, we examined the rest
23 (96\%, nine female) infants with Down syndrome.

There were no significant differences in the birth weights or postconceptional age (PCA) between the two groups, control: $\mathrm{n}=23$ (nine female), $2,551 \pm 615 \mathrm{~g}$ and $39.8 \pm 1.0$ weeks; Down syndrome: $\mathrm{n}=23$ (nine female), 2,555 \pm 535 $\mathrm{g}$ and $40.1 \pm 1.3$ weeks. Table 1 shows the mean CLs and IPLs in the two groups of neonates with and without Down syndrome. As shown in Table 1, wave I CL was longer in ABRs of infants with Down syndrome than control newborns, while wave V CL and the wave III-V and wave I-V IPLs were shorter in ABRs of infants with Down syndrome than control newborns.

\section{Discussion}

The current results in ABRs indicate the presence of developmental anomaly of the inner ear in newborns with Down syndrome [9-11]. These have been suggested to be due to the developmental brain abnormalities associated with central myelination delay and the malformed bone islands of lateral semicircular canal, narrow internal auditory canals and cochlear nerve canal stenosis which have been observed on computed tomography (CT) images in patients with Down syndrome [9]. In addition, the ear dimensions, position and shape in patients with Down syndrome have been observed to be different from those in the sex-, age- and ethnic-matched controls [10], and the cochlear length has been observed to be slightly shorter in temporal bones from patients with Down's syndrome than that in the controls [11]. Therefore, the current results also support these previous observations [9-11].

On the other hand, the current results may be contrary to those in American newborns with Down syndrome by Kit- 
ter et al [7]. They observed the shorter wave III and wave $\mathrm{V}$ CLs and the wave I-III IPL in ABRs for newborns with Down syndrome indicating the presence of anomalies in the lower rather than upper brainstem auditory pathways [7]. The reasons leading to the different results between the two observations are not clear. One may be the small sample size of the both studies (23 and 15 newborns with Down syndrome, respectively). The other reason may be the difference in the test PCA or days to ABRs between the two studies (our study: $40.1 \pm 1.3$ weeks and $20.6 \pm 6.0$ days after delivery; the study by Kitter et al [7]: $37.3 \pm 1.8$ weeks and $3.3 \pm 2.2$ days after delivery; $\mathrm{P}<0.05$ ). All Down syndrome children have been observed to be different degrees in developmental disabilities, developmental delay and developmental brain abnormalities during the developmental periods of their lives $[2,3]$. Therefore, a large study of ABRs examination may be required over time basis during the neonatal period with Down syndrome.

The current results indicate the presence of developmental anomaly of the inner ear in newborns with Down syndrome. However, a larger study is needed.

\section{References}

1. Erenberg A, Lemons J, Sia C, Trunkel D, Ziring P. Newborn and infant hearing loss: detection and intervention.American Academy of Pediatrics. Task Force on Newborn and Infant Hearing, 1998- 1999. Pediatrics. 1999;103(2):527-530.

2. Wisniewski KE. Down syndrome children often have brain with maturation delay, retardation of growth, and cortical dysgenesis. Am J Med Genet Suppl. 1990; 7:274281.
3. Koo BK, Blaser S, Harwood-Nash D, Becker LE, Murphy EG. Magnetic resonance imaging evaluation of delayed myelination in Down syndrome: a case report and review of the literature. J Child Neurol. 1992;7(4):417421.

4. Raut P, Sriram B, Yeoh A, Hee KY, Lim SB, Daniel ML. High prevalence of hearing loss in Down syndrome at first year of life. Ann Acad Med Singapore. 2011;40(11):493-498.

5. Park AH, Wilson MA, Stevens PT, Harward R, Hohler $\mathrm{N}$. Identification of hearing loss in pediatric patients with Down syndrome. Otolaryngol Head Neck Surg. 2012;146(1):135-140.

6. Squires N, Ollo C, Jordan R. Auditory brain stem responses in the mentally retarded: audiometric correlates. Ear Hear. 1986;7(2):83-92.

7. Kittler PM, Phan HT, Gardner JM, Miroshnichenko I, Gordon A, Karmel BZ. Auditory brainstem evoked responses in newborns with Down syndrome. Am J Intellect Dev Disabil. 2009;114(6):393-400.

8. Sato M, Suzuki S. Referral rates and costs of our modified two-step newborn hearing screening program at a Japanese perinatal center. Int J Clin Pediatri. 2014;3:3539.

9. Igarashi M, Takahashi M, Alford BR, Johnson PE. Inner ear morphology in Down's syndrome. Acta Otolaryngol. 1977;83(1-2):175-181.

10. Intrapiromkul J, Aygun N, Tunkel DE, Carone M, Yousem DM. Inner ear anomalies seen on CT images in people with Down syndrome. Pediatr Radiol. 2012;42(12):1449-1455.

11. Sforza C, Dellavia C, Tartaglia GM, Ferrario VF. Morphometry of the ear in Down's syndrome subjects. A three-dimensional computerized assessment. Int J Oral Maxillofac Surg. 2005;34(5):480-486. 